Trabajos Originales

\title{
Caracterización de las pacientes llevadas a cesárea según modelo de Robson y exploración de factores asociados en gestantes atendidas en el hospital Universitario San José de la ciudad de Popayán-Colombia. 1 de enero de 2016 a 30 de junio de 2016.
}

- Alberto Anaya Anichiarico. Md. Residente de III Año de ginecobstetricia. Departamento de ginecología y obstetricia, Facultad de Ciencias de la Salud, Universidad del Cauca.

- Francisco Londoño Villaquiran. Md. Residente de III Año de ginecobstetricia. Departamento de ginecología y obstetricia, Facultad de Ciencias de la Salud, Universidad del Cauca.

- Lorena Pérez Varela Md. Residente de III Año de ginecobstetricia. Departamento de ginecología y obstetricia, Facultad de Ciencias de la Salud, Universidad del Cauca.

- Roberth Ortiz. Martinez. Md. Ginecólogo y obstetra, MSc epidemiologia, profesor titular del Departamento de ginecología y obstetricia, Facultad de Ciencias de la Salud, Universidad del Cauca.

\section{RESUMEN}

INTRODUCCION: a pesar del aumento de la tasa de cesárea no se ha evidenciado una disminución de la morbilidad materna-perinatal, la utilización de modelos como el de Robson permite su auditoria interinstitucional. OBJETIVO: caracterizar las pacientes llevadas a cesárea según el modelo Robson y hacer un análisis exploratorio de sus factores asociados. METODOLOGIA: estudio descriptivo de corte transversal entre el 1 de enero 2016 al 30 de junio 2016, donde se incluyeron todos los nacimientos mayores a 24 semanas, producto único(s), vivo(s), en un Hospital de alta complejidad (Cauca - Colombia) que atiende régimen contributivo y subsidiado. Se tomó el universo, se midieron variables sociodemográficas y biológicas, se aplicó el modelo Robson y mediante regresión logística teniendo en cuenta criterios estadísticos y teóricos, se generaron dos modelos multivariados. RESULTADOS: se analizaron 838 nacimientos, según modelo Robson el grupo más aportante fue el 3 con 236 (28,16\%), y según la contribución de cesáreas en grupo total de atención el 5 fue el más contribuyente (12,17\%). El modelo biológico evidencio significancia en: nuliparidad ORa 3.43; IC95\%; (2,31-5,11); cesárea previa ORa 14.72; IC95\% (7.78-27.85), obesidad ORa 1.66; IC95\% (1.01- 2,74); presentaciones no cefálicas ORa 9.60; IC95\% (3,14-29,31); riesgo intermedio ORa 2,99 IC95\% $(2,01-4,45)$ y alto ORa 7,13; IC95\% (4,13-12,33).

CONCLUSION: El modelo Robson es práctico al aplicar, según este, nuestros resultados son similares a otras instituciones de la misma complejidad. Se encontró significancia en historia de cesárea, obesidad, nuliparidad, presentación diferentes a cefálica y ser clasificada de moderado y alto riesgo.

PALABRAS CLAVE: cesárea, clasificación, riesgo, embarazo.

\section{SUMMARY}

INTRODUCTION: Despite the increase in the cesarean rate, there has been no evidence of a decrease in maternal-perinatal morbidity, the use of models such as that of Robson allows its inter-institutional audit. OBJECTIVE: To characterize the patients carried to cesarean according to the Robson model and to make an exploratory analysis of its associated factors. METHODS: a cross-sectional descriptive study between January 1, 2016 and June 30, 2016, included all births over 24 weeks, single product (s), live(s), in a highly complex Hospital (Cauca - Colombia) That attends contributory and subsidized regime. The universe was taken, sociodemographic and biological variables were measured, the Robson model was applied and by logistic 
regression and taking into account statistical and theoretical criteria, two multivariate models were generated. RESULTS: 838 births were analyzed, according to the Robson model, the most contributing group was 3 with $236(28.16 \%)$, and according to the contribution of cesarean sections in total care group, 5 was the most contributor (12.17\%). The biological model evidenced significance in: nulliparity ORa 3.43; 95\% Cl;( 2.31-5.11); Prior cesarean section ORa 14.72; 95\% Cl (7.78-27.85); obesity ORa 1.66; IC95\% (1.01- 2.74); non-cephalic presentations ORa 9.60; 95\% (Cl 3.14-29.31); moderate risk ORa 2.99 95\% Cl; (2.01-4.45) and high ORa 7.13; 95\% Cl (4.13-12.33). CONCLUSION: The Robson model is practical in applying, according to this, our results are similar to other institutions of the same complexity. Significance was found in history of cesarean section, obesity, nulliparity, non-cephalic presentations and classified as moderate and high risk.

KEYWORDS: cesarean section, classification, risk, pregnancy.

\section{INTRODUCCIÓN}

La operación cesárea se define como "el nacimiento del feto a través de incisiones, en las paredes abdominal (laparotomía) y uterina (histerotomía). Esta definición no incluye la extracción fetal a través de la cavidad uterina en caso de rotura del útero o de un embarazo en esa localización" 1 .Su objetivo es disminuir la morbi-mortalidad materna y perinatal asociada con el momento del nacimiento ${ }^{1}$. Sin embargo, el procedimiento también conlleva riesgos tanto maternos como fetales tanto a corto como a largo plazo ${ }^{2}$.

En los últimos años se ha evidenciado a nivel global un aumento de la tasa de cesárea, en Estados Unidos en 1989 fue cerca del $23 \%$ y para el 2011 del $33 \%{ }^{3}$, para Colombia en 1998, fue del 24,9\% y en 2014 de $46,6 \%$, lo que demuestra un aumento mayor comparado con los Estados Unidos ${ }^{4}$. Para el 2015, la Organización Mundial de la Salud (OMS) consideró que la tasa ideal de cesárea debía ser menor al $15 \%$, y que tasas superiores al $10 \%$ no se asociaban con reducción de la mortalidad materna o de los recién nacidos ${ }^{4-7}$.Sin embargo, la evidencia es escasa al respecto y algunos autores consideran que no se debería establecer una tasa ideal, ya que la cesárea es consecuencia de una decisión clínica basada en valores y condiciones individuales ${ }^{4}$.

El porcentaje de cesárea es cada vez más utilizado en el análisis del desempeño hospitalario por ser un indicador multidimensional que aborda aspectos de adecuación de la atención médica, seguridad del paciente (los riesgos maternos y neonatales relacionados con la sub y sobre utilización) y eficiencia en utilización de los recursos ${ }^{8}$, 9 . En los últimos años, los gobiernos y los profesionales de la salud han expresado su preocupación respecto del incremento en la cantidad de partos por cesárea y las posibles consecuencias negativas para la salud materno infantil ${ }^{10}$. La OMS realizó dos estudios: una revisión sistemática de los estudios disponibles en los que se pretendía determinar esta tasa a nivel de los países y un análisis mundial con los datos más recientes disponibles ${ }^{10-11}$, determinando que una de las causas principales de la dificultad que representa definir la tasa ideal de cesárea a cualquier nivel es la falta de un sistema de clasificación internacionalmente aceptado y fiable que genere datos estandarizados que permita realizar comparaciones entre poblaciones y pueda ser una herramienta para investigar los factores que determinan la tendencia hacia el aumento de cesáreas ${ }^{10}$. Encontró que la clasificación de Robson permite identificar los grupos que más aportan a la cifra global de cesárea, hacer comparaciones en igualdad de condiciones entre instituciones y dentro de la misma institución en el tiempo, y planear intervenciones específicas ${ }^{12-13}$. La utilización de este modelo ha sido fácil de implementar, es reproducible y ha contribuido a lograr efectos positivos de disminución en la proporción de cesáreas sin aumentar la morbimortalidad perinatal ${ }^{12}$ $-15$

Esta clasificación ha ganado importancia en los últimos años en muchos países. Es por ello que ante esta situación surge la necesidad de plantear la presente investigación, que tiene como objetivo : caracterizar las pacientes llevadas a cesárea según el modelo Robson y hacer un análisis exploratorio de sus factores asociados, lo cual permitirá una estandarización al usar este sistema, facilitando comparación de las tasas y análisis de las cesárea y de esta manera configurar estrategias efectivas dirigidas específicamente para optimizar las tasas de cesárea en caso necesario y proporcionar información útil para los proveedores de atención de la salud en el seguimiento y planificación acciones efectivas para reducir estas tasas. 


\section{MATERIALES Y METODOS}

Se realizó un estudio descriptivo de corte transversal en el que se incluyeron todas las gestantes quienes consultaron al servicio de ginecología y obstétrica con embarazo con edad gestacionales mayores a 24 semanas para atención de parto bien sea por vía vaginal o cesárea, con producto único(s), vivo(s) que consultaron desde el 1 de enero de 2016 hasta el 30 de junio de 2016 en un hospital público de alta complejidad, Hospital Universitario San José (HUSJ) de la ciudad de Popayan centro de referencia del departamento del Cauca y suroccidente Colombiano, el cual aporta sus servicios a paciente de régimen contributivo y subsidiado, se tomó todo el universo durante este periodo.

Procedimiento: en mujeres a quienes se le atendió su parto vaginal o cesárea, cuya edad gestacional fue estimada por ecografía del primer trimestre o por examen físico realizado al neonato por el pediatra al momento de nacer, se le solicito autorización para participar en el estudio mediante la firma de consentimiento informado. Una vez esto, se procedió con la entrevista de las pacientes por personal entrenado mediante un cuestionario semiestructurado diseñado por los investigadores, realizado por expertos y ajustado por prueba piloto, además se aplicó el modelo de ROBSON según su estudio original el cual clasifica a las pacientes en 10 grupos $^{12-13}$. Se elaboró una base de datos en Excel, en esta se hizo control de ingreso de datos a través de reglas de validación a fin de garantizar confiabilidad y calidad de la información, posteriormente se llevó al programa STATA 10.0 en el que se efectuó el análisis.

Se midieron variables como la edad materna, edad gestacional la cual se estratifico en pretermino (entre 24 y 36 semanas 6 días), a término 37 hasta fecha probable de parto y posdatal después de esta fecha; zona de residencia (urbana y rural), tipo de unión (estable y no estable), etnia ( blanca y mestiza de referencia y negro indígena como riesgo), tipo de aseguramiento ( contributivo, subsidiado), estrato socioeconómico (de referencia 3 y 4 y de riesgo 1 y 2), control prenatal adecuado ( número de controles mayor de 3 e inicio de control en primer trimestre ), formula obstétrica, amenaza de parto pretérmino en el actual embarazo, planeación de actual embarazo; presencia de curso psicoprofilactico, escolaridad, ingresos del núcleo familiar, peso fetal al nacer, Índice de Masa Corporal (IMC), el cual se definió como normal entre $20-24.9 \mathrm{~kg} / \mathrm{m} 2$, bajo menor a 20 , sobrepeso entre 25 - 29.9, obesidad entre 30 o más, presentación fetal ( cefálico, podálico y otros), se generó una variable según el riesgo obstétrico clasificando a la paciente como de bajo riesgo: aquellas pacientes con transcurso normal del embarazo sin antecedentes o factores de riesgo que pongan en riesgo a gestación; riesgo intermedio: aquellas con alteracion o patología preexistente que pone en riesgo el embarazo pero que no representa en sí una indicación absoluta de cesárea o inducción ( polihidramnios, hipertensión arterial crónica , hipertensión arterial asociada al embarazo, diabetes gestacional , miomatosis, colestasis, riesgo de isoinmunizacion), riesgo alto : enfermedades preexistentes maternas $\mathrm{u}$ otras afecciones obstétricas que estaban presentes y que sugieren la interrupción del embarazo por cesárea o inducción ( infección por $\mathrm{VIH}$, miomectomia, diabetes pregestacional, preeclampsia severa, placenta previa, oligohidramnios severo , RCIU severo), clasificación esta utilizada en un estudio previo 9 .

Para determinar la prevalencia de cesárea se tomó como numerador la población a quien se le practico cesárea por cualquier indicación $y, \quad$ como denominador el total de gestantes atendidas ya sea parto vaginal o cesárea. Las variables se analizaron de forma individual desde el punto de vista exploratorio lo que permitió mirar la normalidad de su distribución e identificar valores extremos y perdidos que pudieron incidir en resultados. Posteriormente se clasificaron las pacientes en los 10 grupos clínicos los cuales son mutuamente excluyentes descritos previamente por Robson ${ }^{13-14}$, se calculó la relación entre las pacientes pertenecientes a cada uno de los grupos de Robson en referencia al total de la población atendida, tomando como numerador el número total de pacientes atendidas pertenecientes a cada grupo y como denominador el número total de pacientes atendidas tanto por parto vaginal como por cesárea, este resultado representa el tamaño relativo de cada uno de los grupos. También se calculó la proporción especifica de cesáreas dentro de cada uno de los grupos, para esto el numerador fue el número de cesáreas realizadas a mujeres pertenecientes a cada grupo y como denominador el número de nacimientos bien sea vaginales o por cesárea pertenecientes a este mismo grupo $\times 100$. También se realizó la contribución que cada grupo hace al porcentaje general de partos, para esto se dividió el número de cesáreas en el grupo por el total de partos atendidos en el periodo, como numerador se encuentra el número total de cesáreas realizadas 
sobre número total de nacimientos vaginales y por cesárea ${ }^{12}$.

Para determinar los posibles factores asociados a cesárea se compararon las distribuciones de las características de interés entre las pacientes que fueron llevadas a cesárea y las que presentaron parto vaginal usando la prueba de chi2 y Fisher según corresponda. Para las variables continuas con distribución normal se utilizó la prueba T de Student, previo análisis de varianza; para variables con distribución no normal se utilizó la $U$ de MannWhitney, previa aplicación de test de normalidad de Shapiro - Wilk. Para el análisis de las posibles variables explicativas de cesárea se realizó un bivariado donde se determinó la fuerza de la asociación por medio del Odds Ratio (OR) y su respectivo intervalo de confianza al $95 \%$ (IC 95\%) entre las diferentes variables generando así una tabla de contingencia. Con base en los hallazgos del bivariado y por medio de regresión logística se generaron 2 modelos multivariados, uno biológico y uno social. Para seleccionar las variables en cada uno de estos modelos se tomaron en cuenta criterios teóricos y estadísticos, para ello se empleó el procedimiento Stepwise con una probabilidad de entrada de 0.2 y de salida 0.15 . Finalmente se realizó diagnóstico de los modelos con el estadístico de Hosmer Lemeshow. ${ }^{16}$

\section{RESULTADOS}

En el periodo del 1 enero 2016 hasta junio 30 del 2016 se atendieron 838 partos de los cuales 445 fueron cesáreas lo cual corresponde a una prevalencia de cesárea del 53.10 \% IC95\% (49.65 - 56.52). El promedio de edad fue de 26 años, El 79,83\% de los embarazos fue a término, el 58,23 \% eran nulíparas, el $21.12 \%$ presentaron antecedente de cesárea previa, el 36 .63 \% estaban en sobrepeso, el 26,61\% se clasificaron como riesgo intermedio y el $19,95 \%$ como de alto riesgo (Tabla 1).

Con respecto a los grupos Robson el de mayor frecuencia fue el grupo 3, con 236 pacientes $(28,16$ $\%$ ). Sin embargo según proporción especifica de cesárea se encontró que los grupos 8,9 y 5 son los que más aportan, según contribución de cesáreas en grupo total de atención el que más aporta es el 5 con un $12.17 \%$ (Tabla 2).

En la tabla número 3 se muestran los OR no ajustados de los posibles variables explicativas de cesáreas al ser no ajustadas pueden estar permeadas por potenciales variables de confusión. En la tabla número 4 y 5 se muestran los modelos desarrollados con sus respetivos OR ajustados, se encontró en el biológico significancia estadística para: antecedente de cesárea previa ORa de 14,72 IC95\% (7,78-27,85), nuliparidad OR 3,43 IC 95\% (2,31-5,11), pacientes catalogadas como riego intermedio ORa de 2,99 con IC95\% (2,01-4,45), riesgo alto ORa 7,13 IC95\%; $(4,13-12,33)$, obesidad ORa 1,66 IC95\% $(1,01-2,74)$ y presentación diferente a cefálico ORa de 9,60 con IC95\% de (3.14-29,31). No se encontró significancia para ninguna de las variables del modelo social.

Finalmente se realizó la evaluación de los modelos con el estadístico de Hosmer- Lemeshow (HL) encontrando para el biológico $\mathrm{HL} \mathrm{CHI2} \mathrm{=} \mathrm{5,74} \mathrm{(GL,} \mathrm{8;}$ $\mathrm{P}=0.67)$ y para el social: $\mathrm{HL} C H \mathrm{~L}=5.51(\mathrm{GL}, 7$; $\mathrm{P}=0.59$ ), con lo que se concluye que los modelos tienen buen ajuste.

\section{DISCUSION}

Durante el primer semestre del 2016 se atendieron 838 nacimientos con una prevalencia de cesárea de $53,10 \%$ la cual comparada con estudios nacionales en centros de alta complejidad como el estudios de Zuleta et al en la ciudad de Medellín y Jiménez et al de la ciudad de Bogotá es mucho mayor ${ }^{12,17}$. El grupo más frecuente según modelo Robson fue el grupo 3 este hallazgo es similar al reportado por Zuleta et al 12 , otros estudios reportan similares hallazgos ${ }^{17}$. No hay estudios que evidencien reportes diferentes. Respecto a la contribución de cesáreas en grupo total de atención se encontró al grupo 5 como el más aportante, hallazgo similar al estudio de Jiménez et al. y al estudio de Vera et al, donde reportan que la historia de cesárea es uno de los grupos que más aportan a realización de cesárea ${ }^{17,18}$, a diferencia del estudio Zuleta et al. que reportan al grupo 10 como el más contribuyente, esta diferencia puede ser explicada ya que en este estudio la tercera parte de los partos atendidos eran pretérmino, sugiriendo que fue el grupo de gestantes que más les consulto en el periodo de intervención a diferencia del presente estudio que los preterminos correspondieron a una proporción menor $(15,27 \%){ }^{12}$

No obstante el hospital presenta las proporciones específicas de cesárea más altas entre las mujeres del grupo 1 al 4 , es decir pacientes nulíparas o multíparas con embarazo a término sin antecedente de cesárea, con feto en cefálica que han iniciado o no trabajo de parto por lo cual este grupo amerita una caracterización más específica para buscar una 
intervención. Haciendo referencia a la contribución de cesárea en el grupo total de atención, el presente estudio encontró, como se mencionó anteriormente, que el grupo 5 fue el que más contribuyó a la prevalencia, resultado similar al estudio de Betran et al donde el grupo 5 fue el mayor contribuyente a la tasa global de realización del procedimiento (26.7\% de la tasa global) ${ }^{19}$.

Así mismo los grupos 2 y 1 son los que seguidamente más aportan partos terminados en cesárea a la tasa global. Estos grupos son aquellos donde las pacientes nulíparas a término que iniciaron o no trabajo de parto, en cefálica terminaron cesárea. Hallazgos similares al estudio chileno de Vera et al donde encontraron que el grupo 1 tuvo un porcentaje importante de aporte al incremento de este procedimiento $^{18}$. Correlacionando esto con que la gran mayoría de pacientes son consideradas de intermedio y alto riesgo según nuestro estudio, nos hace sugerir que este grupo de pacientes posiblemente es el que más complicaciones y comorbilidades como preeclampsia severa o RCIU severo, consulta a nuestro hospital, así como pacientes con más riesgo de desproporción feto pélvica que presentan distocias durante un trabajo de parto ya instaurado.

Las mujeres clasificadas en el grupo 6 y 7 las cuales corresponden a feto en podálica y las correspondientes a embarazo gemelar dentro del grupo 8 tienen una mayor probabilidad de cesárea, de igual forma las clasificadas en grupo 9 que son aquellas con feto en transversa de por si tienen indicación obstétrica para evacuación por vía alta. El número de pacientes que hacen parte de estos grupos es la menor proporción, encontrando que el aporte a la tasa global de cesáreas es inferior en comparación a los demás grupos con menos riesgo de terminar en cesárea mostrando solo un $3.8 \%$ de contribución a la tasa global inmodificable.

Al realizar los modelos multivariados se encontró que el modelo social no mostro ninguna variables significativa hallazgo similar al estudio de Maso et al ${ }^{9}$. . Por otra parte encontramos en el modelo biológico que la edad gestacional no fue asociada en forma significativa con cesárea, lo cual es contrario en comparación al modelo 1 del estudio europeo realizado por Maso et al, donde reportan alta asociación con el parto prematuro9. En nuestro estudio observamos que el no tener parto previo tiene una asociación positiva, mientras que dicho estudio no vario significativamente entre nulíparas y multíparas. Con respecto a la historia de cesárea, obesidad, presentación diferentes a cefálico, clasificación de riesgo intermedio y alto se encontraron similares resultados ${ }^{9}$.

Dentro de las fortalezas tenemos una adecuado tamaño de muestra, no presentó perdida de datos, todas los nacimientos en esta periodo fueron tomados, el análisis multivariado se realizó ajuste mediante regresión logística lo cual permitió controlar confusores; dentro de las limitaciones del estudio encontramos que no existe un consenso sobre los diagnósticos finales en las historias clínicas, lo que hace necesario una estandarización para la fácil recolección de datos y que no existan confusiones a la hora de clasificar las pacientes en su debido grupo Robson. De igual forma la falta de información algunas historias clínicas, sobre todo la información de conductas clínicas tomadas, no obstante, la disponibilidad de registros electrónicos mejora la calidad de los datos.

Conclusión:

El modelo Robson es fácil de aplicar, según este, nuestros resultados son similares a otras instituciones de la misma complejidad. Se encontró significancia en historia de cesárea, obesidad, nuliparidad, presentación diferentes a cefálica y ser clasificada de moderado y alto riesgo.

Finalmente los autores declaran que el presente estudio se realizó con recursos propios y no tener ningún conflicto de intereses.

\section{BIBLIOGRAFÍA}

1- Cunningham, G., Mac Donald, P. C., Gant, N. F., Leveno, J. K., Glistrap, C. L., Hankins, D. V., \& Clark CS (2002). Williams Obstetricia. Vol 23.; 1992.

2- Fecopen F, O'Neill SM, Agerbo E, et al. [Risk factors for ectopic pregnancy. Case-control study]. Obstet Gynecol. 2014;388(6):815-820. doi:10.1111/1471-0528.12165.

3- Hamilton, B. E., Hoyert, D. L., Martin, J. A., Strobino, D. M., \& Guyer B. Annual summary of vital statistics: 2010-2011. Pediatrics. 2013;131:548558.

4- Rubio, J. et al. Racionalización del uso de la cesárea en Colombia. Consenso de la Federación Colombiana de Obstetricia y Ginecología (Fecolsog) y la Federación Colombiana de Perinatología (Fecopen).itle. Rev Colomb Obstet Ginecol. 2014; 65(2).

5- Gibbons, L., Belizán, J. M., Lauer, J. A., Betrán, A. P., Merialdi, M., \& Althabe F. The global numbers and costs of additionally needed and unnecessary 
caesarean sections performed per year: overuse as a barrier to universal coverage. World Heal Rep. 2010; 30:1-31.

6- Moore, B., Appropriate technology for birth. The Lancet. 1985; 2:436-437.

7- Karim, F. et al. Trends and determinants of caesarean section. Surg Pakistan. 2011; 16:1.

8- Morera M. Variabilidad en la tasa de cesáreas entre hospitales públicos de Costa Rica. Rev Chil Obstet Ginecol. 2013;78(2):119-125.

9- Maso, G. et al. The application of the Ten Group classification system (TGCS) in caesarean delivery case mix adjustment. A multicenter prospective study. PLoS One. 2013;8(6).

10- Declaración de la Organización Mundial de la Salud sobre tasas de cesárea.; 2014. OMS. Hallado en: http://www.scielo.org.co/pdf/rcog/v65n2/v65n2a05 .pdf. Acceso el 12 de mayo de 2017.

11- Betran, AP. et al. A systematic review of the Robson classification for caesarean section: what works, doesn't work and how to improve it. PLoS One. 2014; 9(6): e97769

12- Zuleta, J., Quintero, F. and AMQ-C. Aplicación del modelo de Robson para caracterizar la realización de cesáreas en una institución de tercer nivel de atención en Medellín, Colombia. Estudio de corte transversal. Rev Colomb Obstet Ginecol. 2013; 64(2):90-99.
13- Robson, MS. Classification of caesarean sections. Fetal Matern Med Rev.2001; 12:23-39.

14- Robson MS. Can we reduce the caesarean section rate? Best Pr Res Clin Obs Gynaecol. 2001; 15:179-194.

15- Robson MS and Scudamore IW WS. Using the medical audit cycle to reduce cesarean section rates. Am J Obs Gynecol. 1996; 174:199-205.

16- Hosmer, David W.; Lemeshow, Stanley (2013). Applied Logistic Regression. New York: Wiley. ISBN 978-0-470-58247-3.

17- Jiménez, D., et al. "Tasa de cesáreas por grupos de Robson en una institución de mediana complejidad de la ciudad de Bogotá, 2012-2014." Revista Colombiana de Obstetricia y Ginecología 67.2 (2016).

18- Vera P.G Claudio, Correa B Raimundo, Neira M Jorge, Rioseco R Alonso, Poblete L Andrés. Utilidad de la evaluación de 10 grupos clínicos obstétricos para la reducción de la tasa de cesárea en un hospital docente. Rev. chil. obstet. ginecol. 2004 ; 69( 3 ): 219-226

19- Betrán, AP., et al. "WHO global survey on maternal and perinatal health in Latin America: classifying caesarean sections." Reproductive health 6.1 (2009): 18. 
Tabla N¹. Características basales de las pacientes que presentaron parto vaginal o cesárea en el hospital universitario san José Popayán, Enero a Junio de 2016

\begin{tabular}{|c|c|}
\hline VARIABLE & FRECUENCIA (\%) \\
\hline Edad media (+- DS) & $26,17(+-7,21)$ \\
\hline Menor o igual a 18 años & $121(14.44)$ \\
\hline (19-34 años) & $572(68.26)$ \\
\hline 35 o más años & $145(17.30)$ \\
\hline \multicolumn{2}{|l|}{ Edad Gestacional } \\
\hline Termino & $669(79,83)$ \\
\hline Pretérmino & $128(15,27)$ \\
\hline Posdatal & $41(4,89)$ \\
\hline \multicolumn{2}{|l|}{ Historia de Partos } \\
\hline Al menos 1 parto & $350(41.77)$ \\
\hline No partos & $488(58.23)$ \\
\hline \multicolumn{2}{|l|}{ Historia Cesáreas } \\
\hline No historia & $661(78.88)$ \\
\hline Al menos 1 cesárea & $177(21.12)$ \\
\hline \multicolumn{2}{|l|}{ APP en actual embarazo } \\
\hline Sin antecedente & $807(96.30)$ \\
\hline Al menos 1 antecedente & $31(3,70)$ \\
\hline \multicolumn{2}{|l|}{ Índice de Masa Corporal $\left(\mathrm{Kg} / \mathrm{m}^{2}\right)$} \\
\hline Adecuado (20-24.9) & $324(38,66)$ \\
\hline Bajo $(<20)$ & $46(5,49)$ \\
\hline Sobrepeso (25-29.9) & $307(36,63)$ \\
\hline Obesidad (30 o más) & $161(19,21)$ \\
\hline \multicolumn{2}{|l|}{ Estrato } \\
\hline Medio (estrato 3 y 4 ) & $100(11,93)$ \\
\hline Bajo (estrato 1 y 2) & $738(88.07)$ \\
\hline \multicolumn{2}{|l|}{ Riesgo } \\
\hline Bajo & $473(56,44)$ \\
\hline Medio & $223(26,61)$ \\
\hline Alto & $142(19,95)$ \\
\hline \multicolumn{2}{|l|}{ Presentación } \\
\hline Cefálico & $792(94,51)$ \\
\hline Podálico y otros & $46(5,49)$ \\
\hline \multicolumn{2}{|l|}{ Planeación embarazo actual } \\
\hline Si & $67(8.00)$ \\
\hline
\end{tabular}




\begin{tabular}{|c|c|}
\hline No & $771(92,00)$ \\
\hline \multicolumn{2}{|l|}{ Control prenatal adecuado } \\
\hline Adecuado & $515(61,46)$ \\
\hline No adecuado & $323(38,54)$ \\
\hline \multicolumn{2}{|l|}{ Curso psicoprofilactico } \\
\hline $\mathrm{Si}$ & $35(4,18)$ \\
\hline No & $803(95,82)$ \\
\hline \multicolumn{2}{|l|}{ Etnia } \\
\hline Blanca-Mestiza & $697(83,17)$ \\
\hline Indígena-Negra & $141(16,83)$ \\
\hline \multicolumn{2}{|l|}{ Residencia } \\
\hline Urbana & $448(53,46)$ \\
\hline Rural & $390(46,54)$ \\
\hline \multicolumn{2}{|l|}{ Estado civil } \\
\hline Estable & $612(73,03)$ \\
\hline No estable & $226(26,97)$ \\
\hline \multicolumn{2}{|l|}{ Aseguramiento } \\
\hline Contributivo & $143(17.06)$ \\
\hline Subsidiado & $695(82,94)$ \\
\hline \multicolumn{2}{|l|}{ Escolaridad } \\
\hline$>0=9$ años & $483(57,64)$ \\
\hline$<9$ años & $355(42,36)$ \\
\hline \multicolumn{2}{|l|}{ Ingresos en núcleo familiar } \\
\hline$<1$ smlv & $652(77,80)$ \\
\hline$>1 \mathrm{smlv}$ & $186(22,20)$ \\
\hline Peso al nacer (media +- DS) & $3009.96+/-599.68$ gramos \\
\hline 2500-3999 & $692(82.58)$ \\
\hline $1500-1499$ & $105(12,53)$ \\
\hline$<1500$ & $22(2,63)$ \\
\hline$>4000$ & $19(2,27)$ \\
\hline
\end{tabular}

Fuente: Datos propios del estudio.

Los valores se expresan $\mathbf{n}(\%)$

DS: Desviación Estándar

Smlv: Salario mínimo legal vigente 
Tabla N². Caracterización modelo Robson Hospital Universitario San José Popayán, enero a junio de

2016.

\begin{tabular}{|c|c|c|c|}
\hline Grupos & $\begin{array}{l}\text { Tamaño de } \\
\text { grupo }\end{array}$ & $\begin{array}{l}\text { Proporción } \\
\text { especifica cesáreas }\end{array}$ & $\begin{array}{l}\text { Contribución } \\
\text { cesárea en } \\
\text { grupo total de } \\
\text { atención (\%) }\end{array}$ \\
\hline $\begin{array}{l}\text { 1- Mujeres nulíparas con embarazo simple, } \\
\text { feto en cefálica, EG mayor o igual a } 37 \\
\text { semanas en trabajo de parto espontáneo. }\end{array}$ & $222(26,49)$ & $75 / 222(33,78 \%)$ & 8,94 \\
\hline $\begin{array}{l}\text { 2-Mujeres nulíparas con embarazo simple, } \\
\text { feto en cefálica, EG mayor o igual a } 37 \\
\text { semanas a quienes se les realizó inducción } \\
\text { del trabajo de parto o se les practicó cesárea } \\
\text { antes del trabajo de parto }\end{array}$ & $95(11,33)$ & $94 / 95$ (98,94\%) & 11,21 \\
\hline $\begin{array}{l}\text { 3-Mujeres multíparas sin cicatriz uterina } \\
\text { previa, con un embarazo simple, feto en } \\
\text { cefálica, EG mayor o igual a } 37 \text { semanas y } \\
\text { trabajo de parto espontáneo. }\end{array}$ & $236(28,16)$ & $36 / 236(15,25 \%)$ & 4,29 \\
\hline $\begin{array}{l}\text { 4-Mujeres multíparas sin cicatriz uterina } \\
\text { previa, con un embarazo simple en cefálica, } \\
\text { EG mayor o igual a } 37 \text { semanas y se les } \\
\text { realizó inducción del trabajo de parto o se les } \\
\text { practicó cesárea. }\end{array}$ & $58(6,92)$ & $57 / 58(98,27 \%)$ & 6,80 \\
\hline $\begin{array}{l}\text { 5-Todas las mujeres multíparas con } \\
\text { antecedente de al menos una cicatriz uterina, } \\
\text { feto en cefálica, con EG mayor o igual a } 37 \\
\text { semanas }\end{array}$ & $103(12,29)$ & 102/103(99,02\%) & 12,17 \\
\hline $\begin{array}{l}\text { 6-Todas las nulíparas con embarazo simple } \\
\text { en presentación podálica }\end{array}$ & $4(0,47)$ & $3 / 4(75 \%)$ & 0,35 \\
\hline $\begin{array}{l}\text { 7-Todas las multíparas con embarazo simple, } \\
\text { en presentación podálica, con o sin } \\
\text { antecedente de cicatriz uterina. }\end{array}$ & $18(2,14)$ & 15/18 (83,33\%) & 1,78 \\
\hline $\begin{array}{l}\text { 8-Todas las mujeres con embarazo múltiple, } \\
\text { con o sin antecedente de cicatriz uterina. }\end{array}$ & $9(1,07)$ & $9 / 9(100 \%)$ & 1,07 \\
\hline $\begin{array}{l}\text { 9-Todas las mujeres con embarazo simple, en } \\
\text { situación transversa u oblicua, con o sin } \\
\text { antecedente de cicatriz uterina. }\end{array}$ & $5(0,59)$ & $5 / 5(100 \%)$ & 0,59 \\
\hline $\begin{array}{l}\text { 10-Todas las mujeres con embarazo simple, } \\
\text { feto en cefálica, EG de } 36 \text { semanas o menos, } \\
\text { con o sin antecedente de cicatriz uterina. }\end{array}$ & $88(10,50)$ & 49/88 (55,68\%) & 5,84 \\
\hline TOTAL & 838 & $445 / 838$ & 53,10 \\
\hline
\end{tabular}

Fuente: Datos propios del estudio. 
Tabla $N^{\circ}$ 3. Análisis bivariado de posibles factores asociados a cesárea, Hospital Universitario San José Popayán, enero a junio de 2016

\begin{tabular}{|c|c|c|c|c|}
\hline Variable & $\begin{array}{c}\text { Partos } \\
\text { vaginales } \\
(n=393)\end{array}$ & $\begin{array}{c}\text { cesáreas } \\
(n=445)\end{array}$ & OR & IC 95\% \\
\hline \multicolumn{5}{|l|}{ Edad } \\
\hline$(19-34)$ & 264 (67.18) & $308(69.21)$ & Ref. & \\
\hline$(<=18)$ & $66(16,75)$ & $55(12.36)$ & 0.71 & $0.47-1.07$ \\
\hline$(350>)$ & $63(16.02)$ & $82(18.43)$ & 1.11 & $0.76-1.64$ \\
\hline \multicolumn{5}{|l|}{ Edad Gestacional } \\
\hline Termino & 323 (82.19) & $346(77.75)$ & Ref. & \\
\hline Pretérmino & 45 (11.45) & $83(18.65)$ & 1,72 & $1,14-2,61$ \\
\hline Postdatal & $25(6,36)$ & $16(3.60)$ & 0,59 & $1,29-1,18$ \\
\hline \multicolumn{5}{|l|}{ Historia partos } \\
\hline Al menos 1 parto & $206(52.42)$ & $144(32,36)$ & Ref. & \\
\hline No partos & $187(47,58)$ & $301(67,64)$ & 2,3 & $1,72-3,07$ \\
\hline \multicolumn{5}{|l|}{ Historia Cesáreas } \\
\hline No historia & 381 (96.95) & $280(62,92)$ & Ref. & \\
\hline Al menos 1 cesárea & $12(3,05)$ & $165(37.08)$ & 18,7 & $10,13-37,58$ \\
\hline \multicolumn{5}{|c|}{ Antecedente APP emb. actual } \\
\hline Sin antecedente & $383(97.46)$ & $424(95.28)$ & Ref. & \\
\hline Al menos 1 antecedente & $10(2.54)$ & $21(4.72)$ & 1.89 & $0,84-4,56$ \\
\hline \multicolumn{5}{|c|}{ Índice de Masa Corporal $\left(\mathrm{Kg} / \mathrm{m}^{2}\right)$} \\
\hline Adecuado (20-24.9) & $170(43,26)$ & $154(34,61)$ & Ref. & \\
\hline Bajo $(<20)$ & $30(7,63)$ & $16(3.60)$ & 0,58 & $0,28-1,16$ \\
\hline Sobrepeso (25-29.9) & $135(34,35)$ & $172(38,65)$ & 1,4 & $1,01-1,94$ \\
\hline Obesidad (30 o más) & $58(14.76)$ & $103(23,15)$ & 1,96 & $1,30-2,95$ \\
\hline \multicolumn{5}{|l|}{ Estrato } \\
\hline Medio (3 y 4) & $37(9,41)$ & $63(14,16)$ & Ref. & \\
\hline Bajo (1 y 2) & $356(90,59)$ & $382(85.84)$ & 0,63 & $0,39-0,98$ \\
\hline \multicolumn{5}{|l|}{ Riesgo } \\
\hline Bajo & $287(73.03)$ & $186(41,80)$ & Ref. & \\
\hline Medio & $81(20,61)$ & $142(31.91)$ & 2.70 & $1,92-3,81$ \\
\hline Alto & $25(6,36)$ & $117(26.29)$ & 7.22 & $4,44-10,03$ \\
\hline \multicolumn{5}{|l|}{ Presentación } \\
\hline Cefálico & $389(98,98)$ & $403(90,56)$ & Ref. & \\
\hline Podálico y otros & $4(1,02)$ & $42(9,44)$ & 10.13 & $3,62-39.20$ \\
\hline
\end{tabular}




\begin{tabular}{|c|c|c|c|c|}
\hline \multicolumn{5}{|c|}{ Planeación embarazo actual } \\
\hline Si & $27(6.87)$ & $40(8.99)$ & Ref. & \\
\hline No & $366(93,13)$ & $405(91,01)$ & 0,74 & $0,43-1,27$ \\
\hline \multicolumn{5}{|c|}{ Control prenatal } \\
\hline Adecuado & $231(58,78)$ & $284(63,82)$ & Ref. & \\
\hline No adecuado & $162(41,22)$ & $161(36,18)$ & 0.80 & $0.60-1.07$ \\
\hline \multicolumn{5}{|c|}{ Curso psicoprofilactico } \\
\hline Si & $18(4.58)$ & $17(3.82)$ & Ref. & \\
\hline No & $375(95,42)$ & $428(96,18$ & 1,2 & $0,57-2,53$ \\
\hline \multicolumn{5}{|l|}{ Etnia } \\
\hline Banca-mestiza & $331(84,22)$ & $366(82,25)$ & Ref. & \\
\hline Indígena-negra & $62(15,78)$ & $79(17,75)$ & 1,15 & $0,78-1,68$ \\
\hline \multicolumn{5}{|l|}{ Residencia } \\
\hline Urbana & $201(51,15)$ & $247(55,51)$ & Ref. & \\
\hline Rural & $192(48,85)$ & $198(44,49)$ & 0,83 & $0,63-1,11$ \\
\hline \multicolumn{5}{|l|}{ Estado civil } \\
\hline Estable & $293(74,55)$ & $100(25,45)$ & Ref. & \\
\hline No estable & $319(71,69)$ & $126(28,31)$ & 1,15 & $0,84-1,59$ \\
\hline \multicolumn{5}{|l|}{ Aseguramiento } \\
\hline Contributivo & $61(15,52)$ & $82(18,43)$ & Ref. & \\
\hline Subsidiado & $332(84,48)$ & $363(81,57)$ & 0.81 & $0,55-1,18$ \\
\hline \multicolumn{5}{|l|}{ Escolaridad } \\
\hline$>0=9$ años & $216(54,96)$ & $267(60,00)$ & Ref. & \\
\hline$<9$ años & $177(45,04)$ & $178(40,00)$ & 0.81 & $0.61-1,08$ \\
\hline \multicolumn{5}{|c|}{ ingresos núcleo familiar } \\
\hline$<1$ smlv & $316(80,41)$ & $336(75,51)$ & Ref. & \\
\hline$>1 \mathrm{smlv}$ & $77(19,59)$ & $109(24,49)$ & 1,33 & $0,94-1,87$ \\
\hline
\end{tabular}

Fuente: Datos propios del estudio.

IC: Intervalo de confianza

OR: Odds Ratio

Ref: Referencia

Smlv: salario mínimo legal vigente 
Tabla $N^{\circ} .4$ modelo biológico de posibles factores asociados a cesárea, Hospital san José Popayán, enero a junio de 2016.

\begin{tabular}{|l|c|c|}
\hline \multicolumn{1}{|c|}{ Variable } & ORa & IC 95\% \\
\hline$<\mathbf{1 8}$ & 0,86 & $0,53-1,39$ \\
\hline$>\mathbf{3 4}$ & 1,13 & $0,69-1,85$ \\
\hline Pretérmino & 1,16 & $0,70-1,92$ \\
\hline Posdatal & 1,18 & $0,57-2,46$ \\
\hline Nuliparidad & 3,43 & $2,31-5,11$ \\
\hline Cesárea previa & 14,72 & $7,78-27,85$ \\
\hline App en actual embarazo & 1,49 & $0,56-3,96$ \\
\hline IMC bajo & 0,64 & $0,30-1,34$ \\
\hline Sobrepeso & 1.30 & $0,88-1,92$ \\
\hline Obesidad & 1,66 & $1,01-2,74$ \\
\hline Riesgo moderado & 2,99 & $2,01-4,45$ \\
\hline Riesgo alto & 7,13 & $4,13-12,33$ \\
\hline podálico y otros & 9,60 & $3,14-29,31$ \\
\hline
\end{tabular}

Modelo 1: edad materna, edad gestacional, partos, historia de cesárea, amenaza de parto pretérmino, índice de masa corporal, riesgo, presentación.

Fuente: Datos propios del estudio

ORa: Odds Ratio ajustado.

IC: Intervalo de Confianza

App: amenaza de parto pretérmino

IMC: índice de masa corporal 
Tabla N.5 modelo social de posibles factores asociados a cesárea, Hospital San José Popayán, enero a junio de 2016.

\begin{tabular}{|l|l|l|}
\hline \multicolumn{1}{|c|}{ Variable } & ORa & IC 95\% \\
\hline Estrato bajo & 1,08 & $0,90-1,30$ \\
\hline Control prenatal no adecuado & 0,87 & $0,65-1,16$ \\
\hline$<9$ años de escolaridad & 0.98 & $0,73-1,31$ \\
\hline$<$ ssmlv & 1,18 & $0,82-1,71$ \\
\hline
\end{tabular}

Modelo 2: Estrato, control prenatal, escolaridad, ingresos familiares

Fuente: Datos propios del estudio

ORa: Odds Ratio ajustado.

IC: Intervalo de Confianza 